\title{
Farewell Editorial
}

\author{
Dear TAAS Community,
}

After more than 6 years, our term as co-Editors-in-Chief of TAAS has come to an end. Since we started this adventure in April 2011, we have worked hard, along with the Editorial Board, to make TAAS an attractive forum for researchers and practitioners in the exciting area of autonomous and adaptive systems.

One key goal that we set at the beginning and have continually worked toward is ensuring that the readers always have access to timely and quality research. This required reducing the turnaround time of the peer-review process as well as the time-to-publication of the articles submitted to TAAS. Along with the hardworking and dedicated editorial board, we have brought the average review turnaround time, that is, the time from paper submission to notification, down to 93 days from the original 180 days when we took over as co-editors in 2011.

Of course, there are cases in which some articles took a longer time to review, due to the difficulty of finding appropriate reviewers, the complexity of the articles to be reviewed, unforeseen unavailability of an $\mathrm{AE}$, and so on. While we have tried to minimize such cases, the problem is inherent in the review process, which relies on the voluntary work of researchers. Nevertheless, we believe we are leaving the journal in good status, and for this we must thank the active participation and collaborations by all the members of the Editorial Board and by the myriad of reviewers who have volunteered their precious time over these years.

A second key objective was to grow and more effectively engage the community, which is very important for improving the quality of papers submitted to TAAS as well as for making sure that submitted papers reflect the interest of our readers and the best research results in the area. To achieve this, we did the following.

- We enlarged and diversified the editorial board-in addition to making sure that it included the necessary expertise to properly handle the review processes for submitted articles, we also made sure that it represented the variety of research communities that are part of the broad area of "autonomous and adaptive systems."

- We proactively contacted the steering and organization committees of some of the major conferences in the area of "autonomous and adaptive systems."

The result of these activities are, on the one hand, in an editorial board that represents areas as diverse as software engineering, distributed and high-performance computing, robotics, multiagent systems, just to mention a few, and in the publication of many special sections that included selections of the best papers appearing in key conferences, such as SASO (IEEE Conference Series Self-adaptive and Self-organizing Systems), SEAMS (Symposium Series on Software Engineering for Adaptive and Self-managing Systems), and ICAC (International Conference on Autonomic Computing).

\section{So long, thank you, and welcome}

We thank all the distinguished researchers who, over the past 6 years, have agreed to serve on the editorial board and to devote their expertise and time to TAAS, and all the anonymous reviewers who have volunteered their service.

2018 Copyright is held by the owner/author(s).

1556-4665/2018/01-ART17

https://doi.org/10.1145/3149484

ACM Transactions on Autonomous and Adaptive Systems, Vol. 12, No. 4, Article 17. Publication date: January 2018. 
We also thank Dr. Nicola Bicocchi, University of Modena e Reggio Emilia, Italy, and Dr. Ivan Rodero, Rutgers University, USA, who during these years have served as Assistant and Information Manager, respectively, and have helped us in keeping the review process of TAAS effective and the online image of TAAS up-to-date.

Finally, we warmly welcome Professor Bashar Nuseibeh, who has been appointed as the new Editor-in-Chief of TAAS, starting October 1, 2017. We have worked with him to ensure a smooth transition, and we have discussed the past, present, and future of TAAS. We are confident that Professor Nuseibeh is the right person to take on the role of Editor-in-Chief of TAAS. He is experienced, motivated, enthusiastic, and has plenty of fresh ideas and projects for TAAS. This is why Editors-in-Chief of journals must cycle: to bring in new ideas and enthusiasm and push the journal into the future. We sincerely wish Professor Nuseibeh the very best.

Manish Parashar and Franco Zambonelli Rutgers University (USA) and University of Modena e Reggio Emilia (Italy) October 2017 Editor-in-Chief 